\title{
False Positive Drug Results by ELISA Associated with Enzymatic Hair Digestion
}

\author{
James Barker ${ }^{1}$, Andrea Petróczi ${ }^{2}$, Declan Naughton $\mathrm{P}^{2}$ and Salah Breidi $\mathrm{E}^{1,3^{*}}$ \\ ${ }^{1}$ School of Pharmacy and Chemistry, Kingston University, UK \\ ${ }^{2}$ School of Life Sciences, Kingston University, UK \\ ${ }^{3}$ DNA legal, part of DNA Worldwide Group, UK
}

Submission: May 05, 2017; Published: June 06, 2017

*Corresponding author: Salah Breidi E, School of Pharmacy and Chemistry, Kingston University, Penrhyn Road, London, UK, Tel: 07590036466; Email: salah@dnalegal.com

\begin{abstract}
Following the experiment that have been designed for immunological screening test followed by a GC-MS confirmation method for the simultaneous analysis of delta-9-tetrahydrocannabinol (9-THC) , 11-nor-9-Carboxy-THC (THC-COOH), 11-Hydroxy-THC (OH-THC), cocaine, benzoylecgonine (BZ) in human hair and avoiding the significant factors responsible for drug degradation by acid and alkali hydrolysis and replace it with enzymatic hair digestion to obtain optimal recovery conditions, that resulted a false positive results after the radioimmunoassay pre-analysis associated with enzymatic hair digestion. Enzymatic hair digestion was used to hydrolyse 18 Turkish samples using proteinase $\mathrm{K}$, Dithiothreitol and Tris $\mathrm{HCl}$ buffer. At the beginning 18 out of 18 samples showed to be screened positive on ELISA, towards the end using a threshold of $0.1 \mathrm{ng} / \mathrm{mg}$ in $50 \mathrm{mg}$ hair, 2 positive results were found in 18 cases. It was revealed that the 2 samples were positive for 9-THC and THC-COOH. Cross reaction lead to false positive results in the pre screening step as a result of the degradation of the antibodies in the precoated ELISA micro plate. Sixteen out of 18 positive detections could not be confirmed by GC-MS. In this study we investigated cause behind the drug screening malfunction.
\end{abstract}

Keywords: Hair analysis; Proteinase K; Dithiothreitol (DTT); False positive; Enzymatic digestion

Abbrevations: BZ: Benzoylecgonine; DTT: Dithiothreitol; TFAA: Triflouroacetic Anhydride; HFBA: Heptafluorobutyric Anhydride; TMS: Trimethylsilyldiethylamine; BSTFA: Bis Trimethylsilyl Trifluoroacetamide; TMCS : Trimethylchlorosilane

\section{Introduction}

In the past, hair analysis techniques were proposed for the detection of toxicities and individual's nutritional status $[1,2]$. In addition, hair screening improves the drug analysis; its characteristics of non-invasiveness; easy storage at room temperature and a rare risk of infection in comparison to blood and urine analysis. Extraction of the analytes from hair is a step prior to the analysis which started by subjection of the hair into past chemical and organic solvents such as hot methanol [3] and the incubation of hair strands in alkaline or acidic medium overnight [4,5]. The solution provided in the hair analysis method should be able to solubilise the drug from the inner core of the hair without causing damage to the analyte. However, the chemical dissociation can cause drug degradation for chemically unstable compounds, such as cocaine, benzoylecgonine, heroine and other ester compounds [6,7]. As a result, the method of choice, which can help in overcoming most of the hair extraction and digestion method weaknesses, can be avoided by a more neutral, body temperature and analyte protective technique which can be found in enzymatic digestion.

Biological digestion method works on destruction of the hair shaft structure and therefore releases the trapped analytes in hair [8]. Enzymes such as proteinase $\mathrm{K}$, protease $\mathrm{E}$ and VIII have been previously used for the hair hydrolysis to hydrolyse the hair samples without the degradation of the unstable drugs that a neutral $\mathrm{pH}$ and under reasonable temperature $\left(37-40{ }^{\circ} \mathrm{C}\right)$ rather than very high temperature is the typical medium for achieving the best recoveries.

The early stages of hair analysis involved qualitative analysis of hair samples [9]. Over the past few years, ELISA has been improved to meet the criteria of hair analysis [10$12]$. ELISA is considered as a screening test for a pre-analysis 
step which can be conducted by one of the spectroscopic or chromatographic techniques [13]. It is reported that enzymatic hair digestion mixture can result in an undesired effect when used simultaneously with ELISA kits [14].

\section{Materials and Methods}

\section{Chemicals and reagents}

Delta-9-tetrahydrocannabinol (9-THC), cocaine and metabolites were obtained from LGC standards (Teddington, UK). Proteinase $\mathrm{K}$ enzyme and HPLC grade pentane were obtained from Sigma Aldrich (Dorset, UK), Dithiothreitol (DTT) and TRIS HCl buffer were purchased from VWR, (Leicestershire, UK). ELISA kits were purchased from Neogen Corporation (KY, USA). Pentane was purchased from Sigma Aldrich (Dorset, UK). HPLC grade hexane, dichloromethane were purchased from Fisher scientific Ltd. (Leicestershire, UK).

\section{Sample preparation}

Hair samples were collected from 18 male Turkish athletes. The samples were decontaminated by rinsing them 3 times with $3 \mathrm{~mL}$ dichloromethane (each time) by vortex mixing for 20 seconds. Decontamination step ensures that hair contaminants such as coloring, sebum, shampoo, etc., are removed from the surface of hair, which may interfere with analysis. The hair samples were cut to ca. $0.5 \mathrm{~mm}$ long segments by scissors. The hair segments were weighed and then subjected to enzymatic digestion using Pro K enzyme.

\section{Digestion and extraction}

Approximately $50 \mathrm{mg}$ of the decontaminated hair was weighed for enzymatic digestion. Fifty mg of DTT (Cleland's reagent) was added and the mixture was conditioned with $1 \mathrm{~mL}$ of TRIS HCl buffer in a glass vial containing the weighed hair. Proteinase $\mathrm{K}$ "also called protease K" enzyme was added in a ratio of $1 \mathrm{mg}$ hair: $1 \mathrm{mg}$ enzyme to the pulverized hair. Along continuous mixing, the mixture was incubated at $37.5^{\circ} \mathrm{C}$ for $50 \mathrm{~min}$. Drug free hair was used as a control.

Spiked hair was digested with enzyme with the concentration of $1 \mathrm{ng} / \mathrm{mg}$ of 9-THC-D3 as internal standards and drug free hair was used as a control. The Drugs were extracted by liquid-liquid extraction (LLE) using $6 \mathrm{~mL}$ of pentane. After vortex mixing and centrifugation ( $1 \mathrm{~min}$ at $238 \mathrm{xg}$ ), the supernatant organic layer was transferred into a fresh glass tube using a glass pasteur pipette (to avoid plastic pipette degradation into the samples) and the hair residue pellet was discarded. The organic layer was mixed with a $25 \mu \mathrm{L}$ aliquot of $1 \mathrm{M} \mathrm{HCl}$ which had been diluted to $1 \%$ in methanol which help prevention of drug evaporation during drying step. The organic layer was evaporated under a stream of nitrogen gas at $50{ }^{\circ} \mathrm{C}$ using a sample concentrator Techno DB-3 (Cambridge, UK). Spiked samples of 0.02-1.50ng/mg were prepared for the 9-THC, cocaine and their metabolites for GC-MS calibration plots. The extracted residue was reconstituted with $60 \mu \mathrm{l}$ and $30 \mu \mathrm{l}$ hexane and screened on ELISA. The remaining residue was transferred to auto sampler vials. Three $\mu \mathrm{L}$ was injected into the GC-MS system for confirmation analysis.

\section{Enzyme linked immunosorbent assay (ELISA)}

The hair samples were first screened by ELISA. All positive samples were then analysed on GC-MS for confirmation. The ELISA screening system consisted of a Cary 50MPR microplate reader (Varian, UK). The enzymatically digested samples were screened by ELISA for the presence of 9-THC, cocaine, benzylcognine, THC$\mathrm{COOH}, \mathrm{OH}-\mathrm{THC}$. The manufacturer's procedure was followed for analysis. The test kit was a qualitative one, designed for screening forensic detection of drugs and metabolites. The ELISA kits operated on the basis of competition between the drug in the hair samples and the conjugate for the antibody binding sites on the pre-coated micro plate. Enzymatically digested samples were loaded into the micro plate wells followed by addition of the drug-enzyme conjugate and incubated at room temperature away from light if possible for $45 \mathrm{~min}$. Unbounded sample and drug-conjugate were used and washing buffer that was prepared with deionised water and the concentrated buffer supplied with the kit. K-blue substrate was added to detect the presence of the drug-enzyme conjugate. After $30 \mathrm{~min}$ of incubation the reaction was stopped by the addition $100 \mu \mathrm{L}$ of $1 \mathrm{~N} \mathrm{H} 2 \mathrm{SO} 4$. The absorbance of each well was measured and read on the micro plate reader at a wavelength of $450 \mathrm{~nm}$. The concentration of the drug in the well is inversely proportional to the color generated.

\section{GC-MS confirmation}

GC-MS confirmations were carried on AGILENT 7890A gas chromatograph, in combination with an AGLINET 5975XL EI/ CI MSD Triple Axis Detector mass spectrometer connected to a 7683B auto sampler (Agilent Ltd., California, USA). The analytical column for GC was a BP-X5 SGE Forte Capillary column (Victoria, Australia) $(30 \mathrm{~m} \times 0.25 \mu \mathrm{m} \times 0.25 \mu \mathrm{ml}, \mathrm{w}, \mathrm{ID})$; a top taper liner was used to minimize flash back and contact with the inlet weldment. The analytes were extracted with $3 \mathrm{~mL}$ of Pentane. A comparison method was conducted between TFAA (triflouroacetic Anhydride), HFBA (Heptafluorobutyric anhydride), TMS (Trimethylsilyldiethylamine), BSTFA (bis(trimethylsilyl) trifluoroacetamide) and bis (trimethylsilyl) trifluoroacetamide (BSTFA) and 1\% trimethylchlorosilane (TMCS). The proper choice of the derivatization agent was used after comparing the sensitivity of each agent towards the drug, and the result of sufficient volatility without the disturbance of the analysis, also reactivity and price of the agents was taken into consideration.

BSTFA+1\% TMCS was the preferred choice for derivatising for cocaine and THC and its metabolites. The extract was dried under nitrogen gas at $\leq 50{ }^{\circ} \mathrm{C}$ and derivatized with $100 \mu \mathrm{L}$ of bis(trimethylsilyl) trifluoroacetamide (BSTFA) and $1 \%$ trimethylchlorosilane (TMCS) and $50 \mu \mathrm{L}$ of ethyl Acetate by heating at $60{ }^{\circ} \mathrm{C}$ for 2 hour. All solutions were evaporated with a gentle stream of nitrogen at $50{ }^{\circ} \mathrm{C}$ until all the liquid dried 
completely to prevent cross-contamination of the derivatizing mixture with the sample. Samples were reconstituted with $60 \mu \mathrm{L}$ hexane and $3.0 \mu \mathrm{L}$ of the sample was auto-injected onto the GC column.

Electron impact (EI) ionisation mode was used accompanied with Helium as a carrier gas and a flow rate of $1.3 \mathrm{~mL} / \mathrm{min}$. Pulsed split less injection was performed at a purge time of 1 min and a purge flow of $53 \mathrm{~mL} / \mathrm{min}$ with an initial pulse pressure of 30 psi reduced to $10 \mathrm{psi}$ after injection; This enhanced the peak shape and sensitivity. The oven temperature for 9-THC was programmed to start from $50{ }^{\circ} \mathrm{C}$, held for $1 \mathrm{~min}$, then increased to $200{ }^{\circ} \mathrm{C}$ at $40{ }^{\circ} \mathrm{C} / \mathrm{min}$, held for $2 \mathrm{~min}$, and increased to $280^{\circ} \mathrm{C}$ at $80^{\circ} \mathrm{C} / \mathrm{min}$, held for $3 \mathrm{~min}$, to a final step of $310^{\circ} \mathrm{C}$ at $80^{\circ} \mathrm{C} / \mathrm{min}$, held for $4 \mathrm{~min}$

The analysis was performed in selected ion monitoring mode (SIM) and between samples, at least one control sample was analysed to monitor cross-contamination. The solvent delay time was $7 \mathrm{~min}$ and THC and cocaine metabolites with a retention times between $8 \mathrm{~min}$ and $13.5 \mathrm{~min}$. The precursor and product ions of the analytes and internal standard (IS) were: 9-THC, $\mathrm{m} / \mathrm{z}$ 371, 386 RT 9.4min; THC-COOH, m/z 371, 473 RT 13.0min; OHTHC, m/z 371, 474 RT 11.8min; 9-THC-D3, m/z 413, 370 RT $10.35 \mathrm{~min}$, cocaine, $\mathrm{m} / \mathrm{z}$ 303, $182 \mathrm{RT}$ 9.9min Benzoylecgonine, $\mathrm{m} / \mathrm{z} 240,361$ RT 10.9min.

\section{Validation}

At the beginning of the validation process, several analytical parameters were first examined. Positive control (LOD: 0.03ng/ $\mathrm{mg}$ for cocaine and $0.015 \mathrm{ng} / \mathrm{mg}$ 9-THC and their metabolites) was conducted for each new plate to ensure the kit was working properly. After this, negative quality controls (hair samples) were run for each series in the same kit. Repeatability of the assay was evaluated by screening spiked hair specimens with a known amount of drugs in the same day. GC-MS validation was achieved by spiking 50mg of blank hair with solutions of the analytes in hexane resulting in calibrator concentrations in the range of $0.04-1.5 \mathrm{ng} / \mathrm{mg}$ for cocaine and metabolites and 0.02-1.50ng/ mg for 9-THC and metabolites and standard curves were linear Table 1: validation results for the main drugs and its metabolites. with R2 values of $0.995,0.989,0.992,0.997$ and 0.976 for 9-THC, THC-COOH, OH-THC, cocaine and benzoylecgonine respectively. Cross contamination was tested by running different blank hair samples. The results showed no peaks in the region of both drug's elution times. The concentrations of the metabolites were calculated by comparison of peak/area ratio of the drug with IS to those from the calibration curve.

\section{Results and Discussion}

Enzymatic digestion of hair could be the method of choice as it overcomes the weaknesses associated with most of the hair digestion methods. Enzymatic digestion can provide a recovery of $93 \%$ for cocaine metabolite, while alkaline hydrolysis causes its chemical destruction and reduce it to less than $60 \%$. The verification of alkaline destruction of psychoactive drugs and its metabolite was achieved by a recovery study that was carried out on reference specimens, where it was reported a loss of drugs due to alkaline digestion with a lower recovery as compared to direct spiked hair extraction. In order to make sure of retaining the maximum amount of drugs, enzymatic digestion was used.

Cross-reactivity was observed for the enzymatically digested metabolite after ELISA screening. The ELISA assay carried out on selected drugs of enzymatically hydrolyzed samples turned out to be positive in 18 cases, higher readings with respect to the values expected were observed for enzymatically digested samples, where DTT and Pro $\mathrm{K}$ were used. Two different experiments constituted of different sample preparation method A (enzymatic digestion) and B (methanol) to evaluate the effect of the digestion method on ELISA. A replicate of $0.6 \mathrm{ng} / \mathrm{mg}$ of cocaine spiked hair sample and a blank sample were screened on ELISA, the comparison of the enzymatically digested sample and blanks with the control showed a very high concentration values. The results of these preliminary tests are illustrated in (Table1 \& Table 2A \& 2B). After many replicates and different digestion methods $A$ and $B$, it appeared that the cross reactivity was the result of the enzymatic digestion. Instead, no significant interferences of method B that were digested differently with the ELISA kit.

\begin{tabular}{|c|c|c|c|c|}
\hline \multirow{3}{*}{ Compound } & \multirow{3}{*}{ Concentration } & \multicolumn{2}{|c|}{ CV (\%) } & \multirow{3}{*}{ Accuracy \% } \\
\hline & & Intraday & Inter Day & \\
\hline & & $\mathrm{N}=6+6+6+6$ & $N=18+18+18+18$ & \\
\hline \multirow{4}{*}{ THC } & 0.02 & 5.3 & 4.2 & 95.3 \\
\hline & 0.1 & 1.2 & 3.6 & 102.5 \\
\hline & 0.5 & 3.1 & 5.5 & 103.5 \\
\hline & 1 & 2.4 & 1.8 & 108.6 \\
\hline \multirow{4}{*}{ THC-COOH } & 0.09 & 7.6 & 13.2 & 97.5 \\
\hline & 0.1 & 8.3 & 13.7 & 101.7 \\
\hline & 0.5 & 8.6 & 1.39 & 107 \\
\hline & 1 & 10.7 & 11.8 & 98.3 \\
\hline
\end{tabular}


Open Access Journal of Toxicology

\begin{tabular}{|c|c|c|c|c|}
\hline \multirow{4}{*}{ OH-THC } & 0.15 & 8.6 & 5.3 & 107.98 \\
\hline & 0.3 & 5.1 & 6.8 & 106.51 \\
\hline & 0.5 & 3.3 & 2.1 & 97.6 \\
\hline & 1 & 4.2 & 7.9 & 98.3 \\
\hline \multirow{4}{*}{ Cocaine } & 0.04 & 6.4 & 5.6 & 95.2 \\
\hline & 0.08 & 3.7 & 2.8 & 97.5 \\
\hline & 1 & 1.2 & 1.7 & 105.3 \\
\hline & 1.5 & 5.7 & 1.5 & 109.4 \\
\hline \multirow{4}{*}{$\begin{array}{c}\text { Benzoylecg- } \\
\text { oe }\end{array}$} & 0.15 & 7.3 & 5.8 & 92.2 \\
\hline & 0.75 & 4.9 & 3.5 & 95.8 \\
\hline & 1 & 2.6 & 6.8 & 104 \\
\hline & 1.5 & 8.1 & 4.6 & 108.3 \\
\hline
\end{tabular}

Table 2A: ELISA Mean recovery of spiked hair sample with different methods before liquid-liquid extraction.

\begin{tabular}{|c|c|c|c|c|}
\hline A & Method & Elisa Recovery (Ng/Ml) & Sd & \%Recovery \\
\hline Solution A & $\begin{array}{c}\text { Hair+Proteinase } \\
\text { K+Dithiothreitol+buffer }\end{array}$ & 1.3 & \pm 0.04 & 216 \\
\hline Solution B & Hair+Methanol+buffer & 0.56 & \pm 0.02 & 93 \\
\hline \multirow{2}{*}{ Blank A } & Hair+Proteinase $\mathrm{K}+$ & 53 & \pm 0.05 & 176 \\
\hline & Dithiothreitol+buffer & & & \\
\hline Blank B & Hair+Methanol+buffer & 0 & \pm 0.002 & 0 \\
\hline
\end{tabular}

Table 2B: ELISA Mean recovery of a spiked hair sample with different methods after liquid-liquid extraction. For composition of individual solutions used see Materials and methods.

\begin{tabular}{|c|c|c|c|c|}
\hline A & Method & Elisa Recovery (Ng/Ml) & Sd & \%Recovery \\
\hline $\begin{array}{l}\text { Solution } \\
\text { A }\end{array}$ & $\begin{array}{c}\text { Hair + Proteinase } \\
\text { K+Dithiothreitol+buffer }\end{array}$ & 0.56 & \pm 0.002 & 93 \\
\hline $\begin{array}{c}\text { Solution } \\
\text { B }\end{array}$ & Hair+Methanol+buffer & 0.52 & \pm 0.004 & 87 \\
\hline Blank A & $\begin{array}{c}\text { Hair+Proteinase } \\
\text { K+Dithiothreitol+buffer }\end{array}$ & 0 & \pm 0.00 & 0 \\
\hline Blank B & Hair+Methanol+buffer & -0.03 & \pm 0.00 & $\sim 0$ \\
\hline
\end{tabular}


According to the ELISA assay theory, the kit operates on the principle of competition between the enzyme conjugate and the drugs in the sample for a specific number of specific antibodies sites on the pre coated assay. Analytes are added at the beginning to the micro plate wells, then followed by the enzyme conjugate and incubated at room temperature. A competition for the binding sites between the analytes and the ELISA conjugate takes place. After incubation the wells are then washed clearing unbounded extra materials. At the end, another incubation period takes place in the presence of the substrate which binds to the enzyme conjugate. As described in the material and method part, the concentration of the drug in the in competitive ELISA is inversely proportional to the color intensity of the plate reader results. For example, the presence of the drug will show in a light color or no color as the concentration of the analyte increases. The absence of the drug analytes in the micro plate will result in a dark color.

DTT is a strong reducing agent which has a redox potential of $-0.33 \mathrm{~V}$ at $\mathrm{pH}$ 7. The reduction of a typical disulfide bond proceeds by two sequential thiol-disulfide exchange reactions $[15,16]$. The same principle apply to the antibodies in the plate, and it proves that the oxidised DTT breaks the disulfide-bonded resulting denature of the disulfide bond-linked heavy and light chains and also at the hinge region of the ELISA's antibodies. Proteinase $\mathrm{K}$ extracted from fungus Tritirachium album. Proteinase $\mathrm{K}$ is commonly used for its broad specificity and its ability to hydrolyze keratin (hair) [17]. The main location of cleavage is the peptide bond bordering to the carboxyl group mostly on the fragment crystallizable region (FC region) of aliphatic and aromatic amino acids with blocked alpha amino group [18]. DTT as a denaturing agent is used to unfold the protein substrates and make them more accessible to the enzyme to attack and break up the hair. After studying the function of both DTT and pro $\mathrm{K}$, it appeared that both have an undesired effect on the particular antibodies. It was found that it has the same effect on

Table 3: Quantitative GC-MS hair results

\begin{tabular}{|c|c|c|c|c|c|c|}
\hline Hair Samples & Gender & 9-Thc Ng/Mg & THC-COOH Ng/Mg & Cocaine And Other Metabolite & Sd & SEM \\
\hline & & & & & \multicolumn{2}{|c|}{$n=3$} \\
\hline H9 & M & 0.3 & 0.1 & ND & 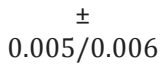 & $0.003 / 0.004$ \\
\hline H 15 & M & 0.2 & ND & ND & $\begin{array}{c} \pm \\
0.003\end{array}$ & 0.002 \\
\hline
\end{tabular}

the ELISA kit. That was proven more when a couple of the micro plate wells were washed with only a solution of Pro K and DTT followed by a blank samples and a spiked samples and all results were with a low absorbance value which prove the theory of the denatured antibodies. As shown in the Figure 1, if the drug is not present more substrate-conjugate binding, in which higher color intensity should be seen under micro plate reader

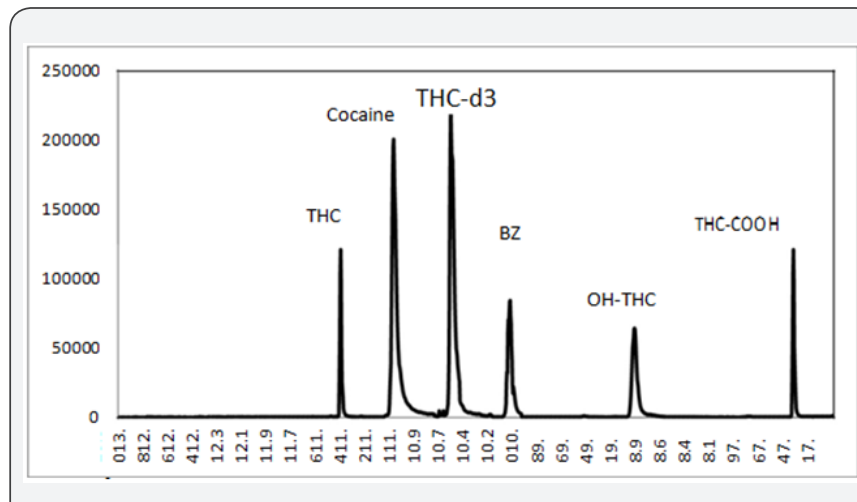

Figure 1: THC, cocaine and their metabolites chromatograms.

On the other hand when the antibodies are been destroyed by DTT and the enzyme, there will be no place for the conjugate or the antigen (drug in this case) to bind and therefore it results in no binding of the substrate- conjugate complex and showing no color but giving the assumption of low absorbance reading of the wells and therefore false positive results (Figure 1). Due to the false positive results that were appearing at the end of the ELISA screening, Liquid-Liquid Extraction was used to clear the DTT and deactivate Pro K enzyme inhibitor. Pentane was used in the LLE due to its low polarity and since DTT is not soluble in it. All the 18 hair samples were clear of the additives and extracted and screened again on ELISA kit (Figure 2). The results this time were different, 2 samples appeared to contain 9-THC on ELISA. GC-MS confirmed sample to contain 9-THC and THC-COOH (Table 3). 

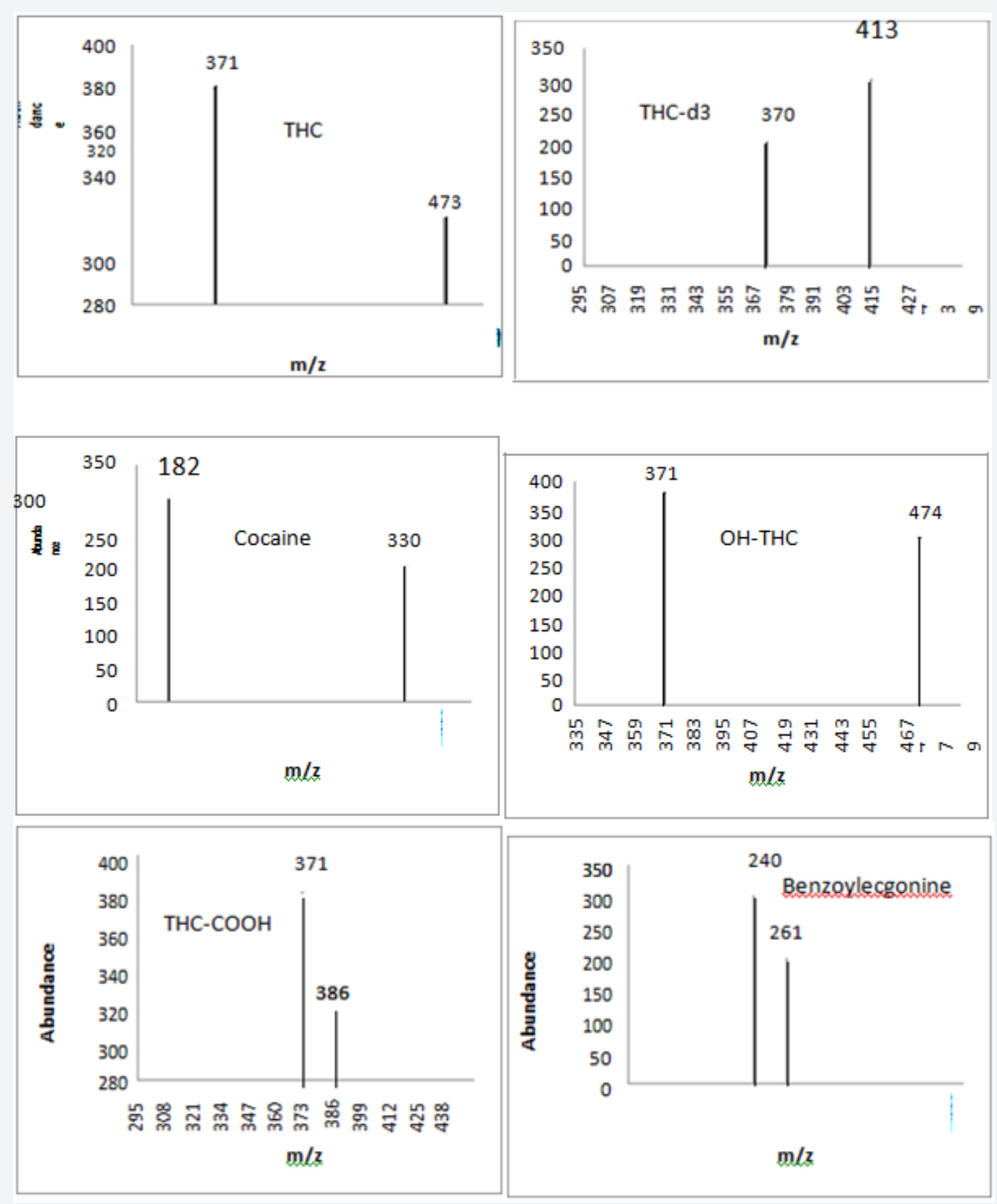

Figure 2: Validation results of main drugs and its metabolism.

\section{Conclusion}

There is an increasing interest in toxicological hair investigation as a marker of individual exposure to many toxicants such as illicit substances. Drug degradation is a serious issue that can be encountered. By using the Pro K for digestion, this protected the drug and enhanced the percent recovery and therefore achieved lower detection limits. This has made the analysis feasible by the extension of a simple highly sensitive and reliable method of detection using gas chromatography mass spectrometry. Hair analysis for many substances can be hard and expensive; also ELISA kits yet didn't expand a general acceptance due to its very narrow selectivity without being able to specify some drugs and metabolites. However, the rapid ELISA screening method can shorten the time and minimise the waste of resources on blank samples, so only positively screened samples continue their way through to confirmation analysis. The enzymatic hair digestion that was performed followed by ELISA screening showed some side effects of ELISA and the enzymatic digestion. False positive samples appeared to be confirmed as negative on ELISA due to DTT and Pro K denaturing the antibodies inside the micro plate wells. LLE and enzyme inhibitors were the key for overcoming the false positive results problem. This key changed the whole conclusion of having 18 drug users lowering it down into only 2 users (Figure $3 \& 4$ ). 


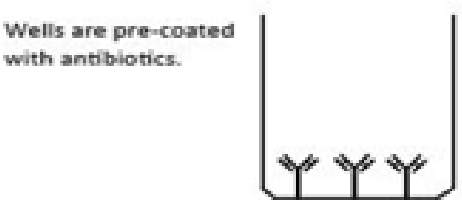

Digested hair

samples or control are added followed by drug enayme conjugrate and incubated at room temperature for 45 minutes in the dark.

Wells are washed using washing buffer 3 times to remove any unbound material.
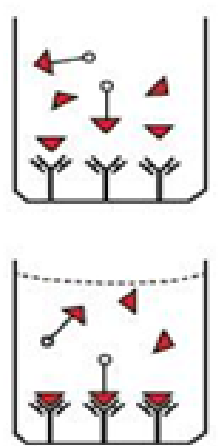

Only the drug samples and the enzyme conjugate remain bound to the antibody in the well

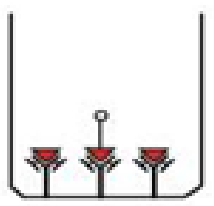

Substrate is added and allowed to bind to the conjugate.

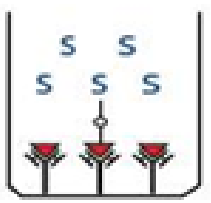

Depending on the amount of substrate bound to conjugate the colour intensity will change. The greater the number of complexes

formed, the higher the absorbance is and consequently the colour intensity

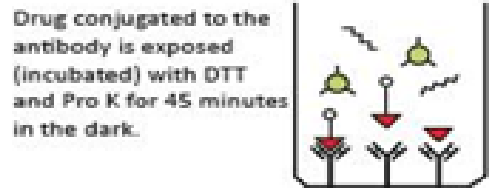

DTT denatures the disulphide bonds between the heavy and light chain of the antibody, and the hinge region. Pro $\mathrm{K}$ cleaves

the peptide bond borderingthe carboxyl eroup.

The antibodies are disrupted and the drug and conjugate are released.
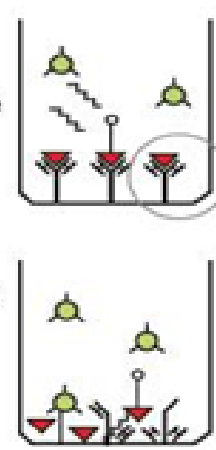

Wells are washed removing all the unbound material.

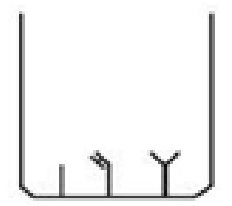

Substrate is unable to bind due to the absence of the conjugate.

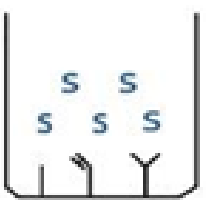

Unbound substrate, results in a very light colour implying a positive result which in fact is a false positive.

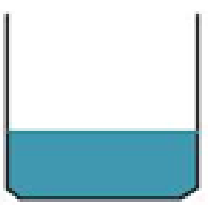

Figure 3: Normal and enzymatically digested samples procedure for competitive direct enzyme-linked immunosorbent assay (ELISA).

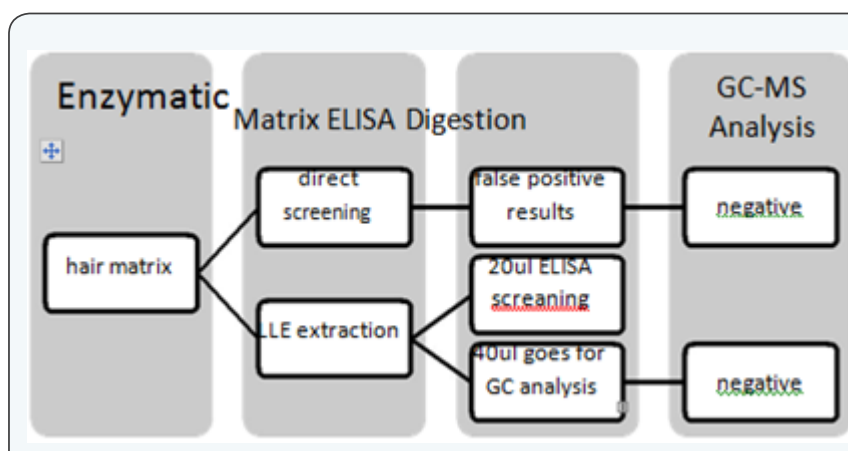

Figure 4: clearing of DTT and Pro $K$ from the hair matrix.

\section{Acknowledgment}

I want to thank Dr. Julian Swinden for technical advice. Also, I would like to thank Mr. Ihsan Sari for data and hair samples collection and the National Council for Scientific Research, CNRS (Lebanon) for the partial funding of my research.

\section{Reference}

1. Bridges JW, Garattini S, Agarwal DK, Barltrop D, Brueton MJ, et al. (1990) Methods to Detect Toxic Effects in Specific Mammalian Organs and Physiological Systems.

2. Baumgartner WA (1994) United States Patent, Ligand Assays of Enzymatic Hair Digests. 
3. Baumgartner AM, Jones PF, Baumgartner WA, Black CT (1979) Radioimmunoassay of Hair for Determining Opiate-Abuse Histories. J Nucl Med 20(7): 748-752.

4. Valente D, Cassini M, Pigliapochi M, Vansetti G (1981) Hair as the sample in assessing morphine and cocaine addiction. Clin Chem 27(11): 1952-1953.

5. Offidani C, Strano Rossi S, Chiarotti M (1993) Improved enzymatic hydrolysis of hair. Forensic Sci Int 63(1-3): 171-174.

6. Nakahara Y (1999) Hair analysis for abused and therapeutic drugs. J Chromatogr B Biomed Sci Appl 733(1-2): 161-180.

7. Offidani C, Carnevale A, Chiarotti M (1989) Drugs in hair: A new extraction procedure. Forensic Sci Int 41(1-2): 35-39.

8. Boumba VA, Ziavrou KS, Vougiouklakis T (2006) Hair as a Biological Indicator of Drug Use, Drug Abuse or Chronic Exposure to Environmental Toxicants. Int J Toxicol 25(3): 143-163.

9. Baumgartner AM, Black CT, Jones PF, Blahd WH (1982) Radio Immuno Assay of cocaine in hair: concise communication. J Nucl Med 23: 790792

10. Verstraete AG (2004) Detection Times of Drugs of Abuse in Blood, Urine, and Oral Fluid. Ther Drug Monit 26(2): 200-205.
11. Jaffee WB, Trucco E, Levy S, Weiss RD (2007) Is this urine really negative? A systematic review of tampering methods in urine drug screening and testing. J Subst Abuse Treat 33(1): 33-42.

12. Spiehler V (2000) Hair analysis by immunological methods from the beginning to 2000. Forensic Sci Int 107(1-3): 249-259.

13. Pragst F, Balikova MA (2006) State of the art in hair analysis for detection of drug and alcohol abuse. Clinica Chim Acta 370(1-2): 1749.

14. M Burrell (1993) Enzymes of molecular biology. The Humana Press,Totowa, NJ, USA, 16: 384.

15. Cleland WW (1964) Dithiothreitol, A New Protective Reagent for SH Groups. Biochemistry 3: 480-482.

16. Edelman GM, Gally JA (1962) The nature of Bence-Jones proteins. Chemical similarities to polypetide chains of myeloma globulins and normal gamma-globulins. J Exp Med 116(2): 207-227.

17. Kraus E, Femfert U (1976) Proteinase K from the Mold Tritirachium album Limber, Specificity and Mode of Action. Hoppe Seylers Z Physiol Chem 357(7): 937-947.

18. Betzel C (1988) Three Dimensional Structure of Proteinase K at 0.15nm Resolution. Eur J Biochem 178(1): 155-171.

\section{Your next submission with Juniper Publishers will reach you the below assets}

- Quality Editorial service

- Swift Peer Review

- Reprints availability

- E-prints Service

- Manuscript Podcast for convenient understanding

- Global attainment for your research

- Manuscript accessibility in different formats

( Pdf, E-pub, Full Text, Audio)

- Unceasing customer service

Track the below URL for one-step submission https://juniperpublishers.com/online-submission.php 